\title{
Thermal and Mechanical Properties of Graphene-Titanium Composites Synthesized by Microwave Sintering
}

\author{
Wen-Zhi Yang ${ }^{1}$ - Wei-Ming Huang ${ }^{1} \cdot$ Zhi-Feng Wang $^{1} \cdot$ Fu-Jun Shang ${ }^{1} \cdot$ Wei Huang ${ }^{1,2} \cdot$ Bao-Yu Zhang ${ }^{1}$
}

Received: 17 February 2015/Revised: 24 April 2015/Published online: 2 June 2016

(c) The Chinese Society for Metals and Springer-Verlag Berlin Heidelberg 2016

\begin{abstract}
The $x$ wt $\%$ graphene-Ti composites $(x=0,0.2,0.3$ and 0.4$)$ were obtained using the powder metallurgy method. The X-ray diffraction results demonstrated that the peak intensity of graphene increased monotonically with increasing graphene content. Furthermore, the number of grain boundary and interface between graphene and matrix increased as graphene increased, which led to a sharp rise of thermal resistances. The thermal conductivity and specific heat capacity of composites initially decreased drastically with addition of graphene, but then increased with increasing graphene content from 0.2 to $0.4 \mathrm{wt} \%$. This phenomenon was connected with the graphene content and the characteristics of Ti matrix (pores, grain boundary and interface between graphene and matrix). The variation of the compressive strength of composites was attributed to the interaction effects of the average grain size of the Ti matrix $\left(d_{\mathrm{m}}\right)$ and the volume fraction $\left(V_{\mathrm{f}}\right)$ and aspect ratio $(\lambda)$ of graphene.
\end{abstract}

KEY WORDS: Graphene; Metal-matrix composites; Thermal properties; Compressive strength

\section{Introduction}

Due to outstanding properties like high thermal conductivity ( $\left.\sim 3000 \mathrm{~W} \mathrm{~m}^{-1} \mathrm{~K}^{-1}\right)$, mechanical strength $(\sim 125 \mathrm{GPa})$ and modulus $(\sim 1.1 \mathrm{TPa})[1]$, the graphene has attracted considerable attentions in recent years. But how to make full use of these remarkable properties of graphene still remains to be a significant issue to material and physics researchers. To incorporate graphene into composites is a possible route to utilize properties into applications.

Available online at http://link.springer.com/journal/40195

Wen-Zhi Yang

yangwenzhith@163.com

1 Ningbo Branch of China Academy of Ordnance Science, Ningbo 315103, China

2 College of Mechanical Engineering and Mechanics, Ningbo University, Ningbo 315103, China
Most studies of graphene incorporation have been concerned about enhancing the properties of polymer matrices, showing a great promise [2,3]. Different from the graphene-polymer composites, the graphene-metal composites were still little discussed owing to the great difficulties in dispersion, fabrication and their unknown interfacial chemical reactions. However, there are a number of recent advances in graphene-metal composites. For example, fewlayer graphene (FLG)-reinforced copper composites were fabricated by spark plasma sintering (SPS) with a FLG volume fraction of $2.4 \mathrm{vol} \%$. The conductance of composites reached $70.4 \%$ of international annealed copper standard (IACS). The yield strength of composites was $376 \mathrm{MPa}$, which was 2.5 times higher than that of copper [4]. Graphene- $\mathrm{Cu}$ nanocomposites foils were synthesized by electrochemical way, which showed a high hardness up to 2.2-2.5 GPa and an elastic modulus to $137 \mathrm{GPa}$, increased by $96 \%$ and $30 \%$ from those of pure $\mathrm{Cu}$, respectively [5]. Graphene-Al composites were obtained by powder metallurgy technique and exhibited greatly improved mechanical properties after adding graphene [6]. 
Furthermore, the mechanical strength of $\mathrm{Mg}-\mathrm{Al}$-graphene nanocomposites proved to be better than $\mathrm{Mg}-\mathrm{Al}$-carbon nanotubes and $\mathrm{Mg}$-ceramic composites [7]. Chen et al. [8] combined liquid-state ultrasonic processing and solid-state stirring into the fabrication of bulk graphene-Mg nanocomposites. After adding $1.2 \mathrm{vol} \%$ graphene nanoplatelets, the microhardness of graphene-Mg composites could be raised to $66 \mathrm{~kg} \mathrm{~mm}^{-2}$, which was $78 \%$ higher than that of pure $\mathrm{Mg}$. In addition, graphene nanoplateletsreinforced AZ61 and AZ31 magnesium alloy was obtained by disintegrated melt deposition (DMD) and powder metallurgy (PM) methods, respectively [9, 10]. Both experimental results demonstrated that the significantly increased microhardness and mechanical strength of composites could be attributed to refined grain size and changes in basal textures by adding graphene.

On the other hand, owing to their excellent properties like high melting point, high tensile strength at room temperature, low density, excellent corrosion resistance and great biocompatibility, $\mathrm{Ti}$ and its alloys are widely used in aerospace, marine and military areas. But due to their vital drawbacks like low thermal conductivity, wear resistance, high cost and low mechanical strength at high temperatures, many researchers put forward carbon materials-Ti composites, such as carbon nanotubes-Ti and carbon fibers-Ti composites, to overcome these disadvantages $[11,12]$. And compared with carbon nanotube and carbon fibers, graphene exhibits even more excellent performance in thermal and mechanical properties. The thermal and mechanical properties of pure $\mathrm{Ti}$ and its alloys may be effectively enhanced by adding a certain amount of graphene. However, the interdisciplinary researches of graphene-Ti composites are rare and only exist in functional materials and theoretical calculation [13, 14]. To our knowledge, the thermal conductivity and mechanical properties of graphene-Ti composites have not been investigated by far.

In the present work, three composites were obtained, namely $0.2 \mathrm{wt} \%$ graphene-Ti, $0.3 \mathrm{wt} \%$ graphene-Ti and 0.4 wt $\%$ graphene-Ti, in comparison with the pure $\mathrm{Ti}$ (0 wt $\%$ graphene-Ti). The objective of this work can be divided into two parts: to fabricate different graphene-titanium composites using the powder metallurgy method and to reveal the correlation between the microstructure and properties.

\section{Experimental}

The $x$ wt $\%$ graphene-Ti composites $(x=0,0.2,0.3$ and 0.4 ) were synthesized by powder metallurgy process. $\mathrm{Ti}$ powder (99.9\%, Xi'an Baode Powder Metallurgy Co. Ltd, China) and graphene platelets (Ningbo Morsh Technology
Co. Ltd, China) with $<10$ layers were adopted as the starting materials. The starting materials were ball-milled by planetary ball mill at a speed of $200 \mathrm{r} / \mathrm{min}$ for $1 \mathrm{~h}$ and then reversed at the same speed for $1 \mathrm{~h}$. One rotation plus one reversion was counted as one circulation. The ball mixing process was continuously performed for six circulations to obtain a homogenous mixture. The ratio of ball to powder was $4: 1$, and the WC ball was $10 \mathrm{~mm}$ in diameter. The mixed powder added with $3 \%$ stearic acid was pressed into cylindrical compacts under a uniaxial pressure of $180 \mathrm{MPa}$. If the stearic acid was $<3 \%$, the mixed powder can hardly be molded. Finally, the $x$ wt $\%$ graphene-Ti composites ( $x=0,0.2,0.3$ and 0.4 ) were obtained by microwave sintering with $2 \mathrm{~kW}$ output power at $1623 \mathrm{~K}$ in low pressure $\left(10^{-3} \mathrm{~Pa}\right)$ for $15 \mathrm{~min}$.

$\mathrm{Ti}$ powder and graphene particle morphologies were observed by scanning electron microscopy (S4800, Hitachi, Japan), and the grain sizes of composites were measured by a computer-controlled optical microscopy (MEF4M, Leica, Germany). The phase of composites was identified by $\mathrm{X}$-ray diffraction (XRD) analysis with $\mathrm{Cu} K \alpha$ radiation (D/max-2500/PC, Rigaku Corporation, Japan). The thermal conductivity of the composites was evaluated by the bulk density, thermal diffusivity and specific heat capacity. The thermal diffusivity of the composites was measured on disk-shaped samples $(10 \mathrm{~mm}$ in diameter and $2.0 \mathrm{~mm}$ in thickness) using a laser flash apparatus (LFA 457, Netzsch, Germany) at room temperature. The density was obtained by the relation $\rho=m / v$, where $m$ is the quality of composites and the $v$ is the volume of disk-shaped composites. The compression tests were carried out to investigate the mechanical properties of the composites using electronic universal testing machines (CMT-4106, Shanghai, China) at room temperature.

\section{Results and Discussion}

The morphologies of the Ti powder (a) and graphene (b) as starting materials are shown in Fig. 1, respectively. The morphology of Ti raw materials is irregular. The particle distribution of $\mathrm{Ti}$ powder ranges from 5 to $45 \mu \mathrm{m}$, with an average particle size of $20 \mu \mathrm{m}$. Graphene has a 2-D planar morphology, and it is much thinner than Ti particles.

The phase structures of composites after microwave sintering were captured by XRD. Figure 2 shows the XRD patterns of graphene, pure $\mathrm{Ti}$ and graphene-Ti composites. All of the major diffraction peaks ((100), (002), (101), (102), (110), (103), (112), (201)) can be indexed as the hexagonal $\mathrm{Ti}$ with the space group P63/mmc (194). The peaks of titanium carbide $\left(\mathrm{Ti}_{8} \mathrm{C}_{5}\right.$, Rhombohedral) can be also observed at $36.1^{\circ}$ and $42.0^{\circ}$ as graphene adding from 0.2 to $0.4 \mathrm{wt} \%$. It is apparent in Fig. 2 that the addition of 

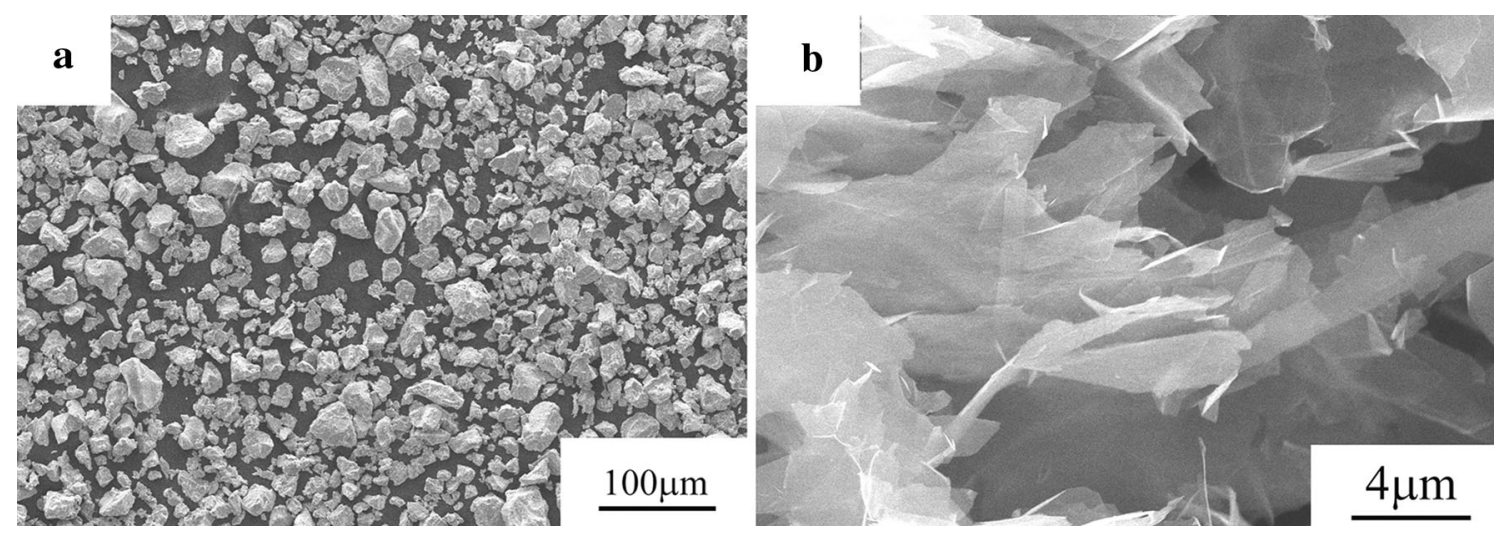

Fig. 1 SEM micrographs of Ti powder $\mathbf{a}$ and graphene $\mathbf{b}$

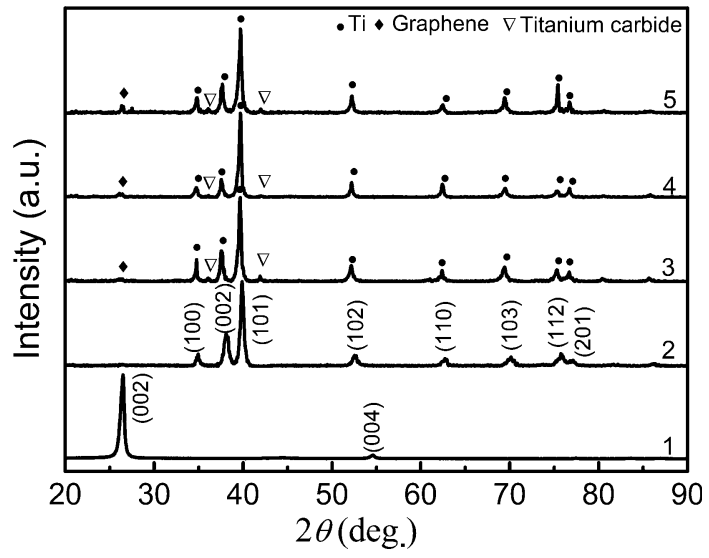

Fig. 2 XRD patterns of graphene (1), pure Ti (2), 0.2 wt\% graphene$\mathrm{Ti}$ (3), $0.3 \mathrm{wt} \%$ graphene-Ti (4) and $0.4 \mathrm{wt} \%$ graphene-Ti (5) composites after microwave sintering

graphene leads to the presence of a new peak which refers to the presence of graphene itself in all graphene-Ti composites. The graphene peak is presented at $26.5^{\circ}$ in all the graphene-containing composites [15]. Meanwhile, the peak intensity of graphene monotonically increases with increasing graphene content. The XRD results indicate that the graphene-Ti composites are obtained by the powder metallurgy method.

The metallographic structures of pure $\mathrm{Ti}(\mathrm{a}, \mathrm{b}), 0.2 \mathrm{wt} \%$ graphene-Ti (c, d), $0.3 \mathrm{wt} \%$ graphene-Ti (e, f) and $0.4 \mathrm{wt} \%$ graphene-Ti composites $(\mathrm{g}, \mathrm{h})$ sintered at $1623 \mathrm{~K}$ for 15 min are shown in Fig. 3, respectively. The grain size of graphene-Ti composites is determined using a linear intercept technique. The average grain size, $G$, is then calculated using the relation [16],

$G=1.5 L /(M N)$,

where 1.5 is a geometry-dependent proportionality constant, $L$ is the total test line length, $M$ is the magnification, and $N$ is the total number of intercepts.
The average grain size of pure $\mathrm{Ti}$ is $215 \mu \mathrm{m}$, while the average grain size of $0.4 \mathrm{wt} \%$ graphene-Ti composites is $7 \mu \mathrm{m}$. The increase of graphene content reduces the grain size obviously, which is due to the nature of graphene. The graphene acts as barriers against the tendency of grain growth, leading to finer grain structure. The grain size decreases with increasing graphene, i.e., the number of grain boundary and interface between graphene and matrix increase with increasing graphene. Besides, the sintering temperature also plays an important role as controlling factor in grain growth process. The following equation confirms this phenomenon [15],

$D=D_{0} \exp (-Q / R T)$,

where $D$ is diffusion coefficient, $D_{0}$ is constant, $Q$ is activation energy, $R$ is Boltzmann's constant, and $T$ is absolute temperature. In addition, observations of the microstructures also show that some pores are visible on the surfaces of the composites. Figure 4 shows the variation of the relative density of graphene-Ti composites with different graphene contents. The relative densities of all composites are above $93 \%$, and the highest relative density just reaches 95\% for $0.4 \mathrm{wt} \%$ graphene-Ti composites. It can be seen that the pores are common in composites, which is consistent with the metallographic analysis.

Figure 5a shows the SEM image of the $0.4 \mathrm{wt} \%$ graphene-Ti composites. The white dots represent carbon elements determined by EDS as shown in Fig. 5b, exhibiting that the carbon is evenly and homogeneously dispersed in $\mathrm{Ti}$ matrix. This suggests that the uniform dispersion of graphene in ball-milled powders can be well obtained by microwave sintering. Furthermore, there are also some pores with homogeneous distribution on the surfaces of composites (Fig. 6). The EDS analysis has been adopted at two different areas, EDS1 and EDS2, and the results are listed in Table 1. It can be seen that the element contents between EDS1 and EDS2 are remarkably different. The $\mathrm{C} / \mathrm{Ti}$ ratio (at.\%) of composites containing pores is 

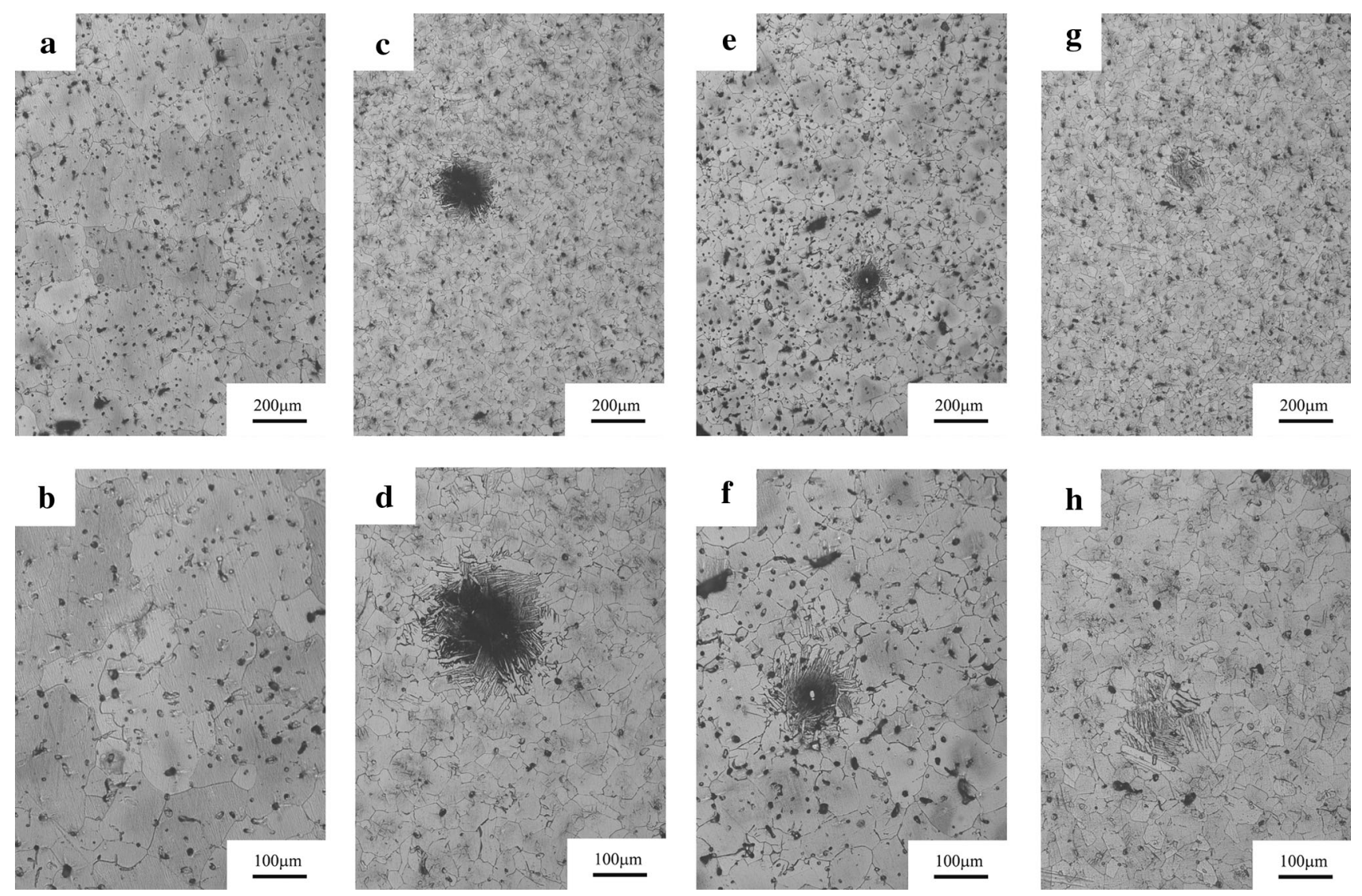

Fig. 3 Metallographic structures of pure Ti a, b, 0.2 wt $\%$ graphene-Ti c,d, 0.3 wt $\%$ graphene-Ti e, f, and 0.4 wt $\%$ graphene-Ti $\mathbf{g}$, h composites sintered at $1623 \mathrm{~K}$ for $15 \mathrm{~min}$

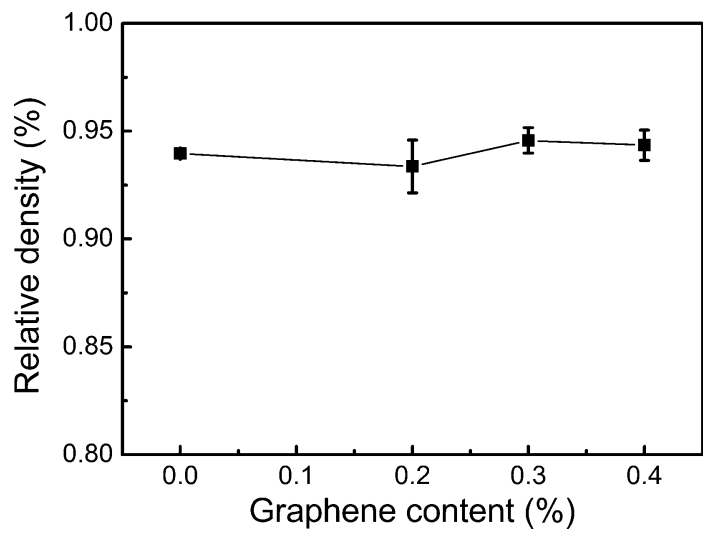

Fig. 4 Relative density of graphene-Ti composites with different graphene contents

61.87/38.13, which is much higher than that on smooth surface $(\mathrm{C} / \mathrm{Ti} \sim 13.29 / 86.71)$. This demonstrates that graphene is prone to agglomerating at pores.

The thermal conductivity and specific heat capacity of graphene-Ti composites with different graphene contents are shown in Fig. 7. The thermal conductivity and specific heat capacity of composites initially decrease drastically with graphene adding, but then increase as graphene increases from 0.2 to $0.4 \mathrm{wt} \%$. The thermal conductivity can be explained by the following equation [17],

$k=\alpha \rho c_{p}$,

where $k$ is thermal conductivity, $\alpha$ is thermal diffusivity, $\rho$ is density, and $c_{\mathrm{p}}$ is specific heat. The increasing graphene concentration is supposed to expect higher thermal conductivity. Since efficient heat propagation in graphene is mainly due to good conduction of phonons through $\mathrm{C}-\mathrm{C}$ bonds, uniform dispersion of graphene in the $\mathrm{Ti}$ matrix may contribute to steady increase in thermal conductivity of the composites. However, the effectiveness of thermal resistance containing contact resistance among graphenes and the interfacial thermal resistance between the graphene and the matrix should all be taken into consideration [18, 19].

In this case, the thermal boundary resistance between the graphene and the matrix should be counted owing to their huge difference in phonon density of states. Assuming randomly oriented graphene and incorporating thermal boundary resistance, the thermal conductivity of composites can be calculated as follows, 

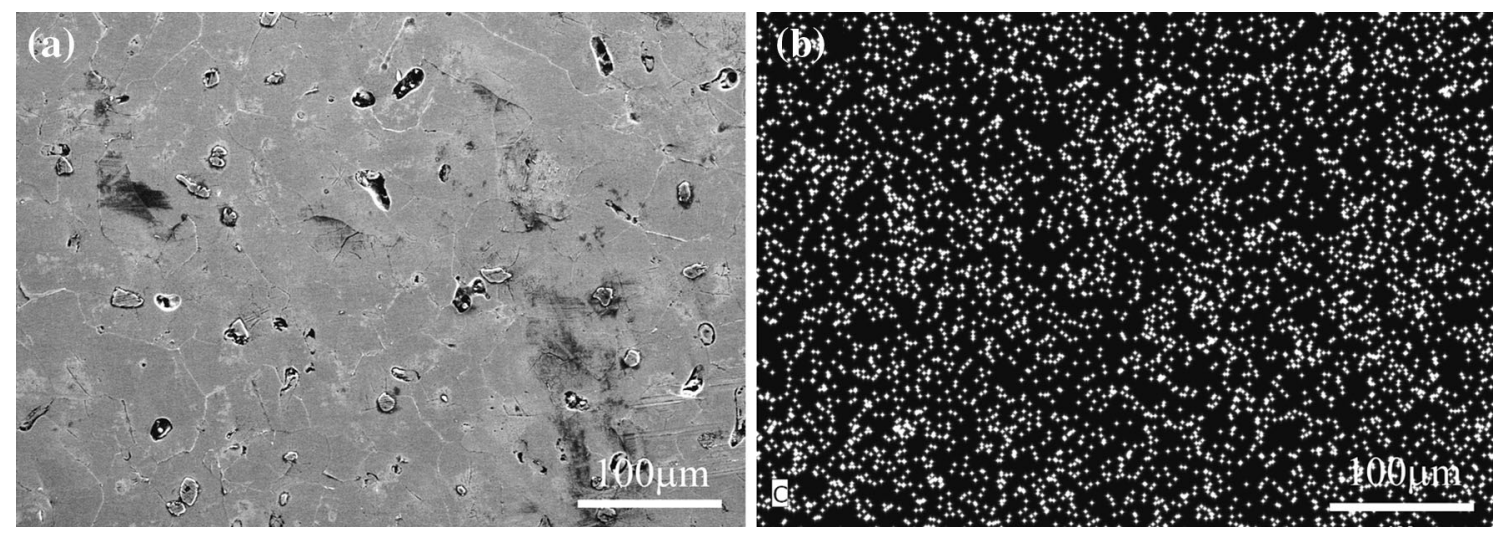

Fig. 5 Surface element analysis of 0.4 wt $\%$ graphene-Ti composites

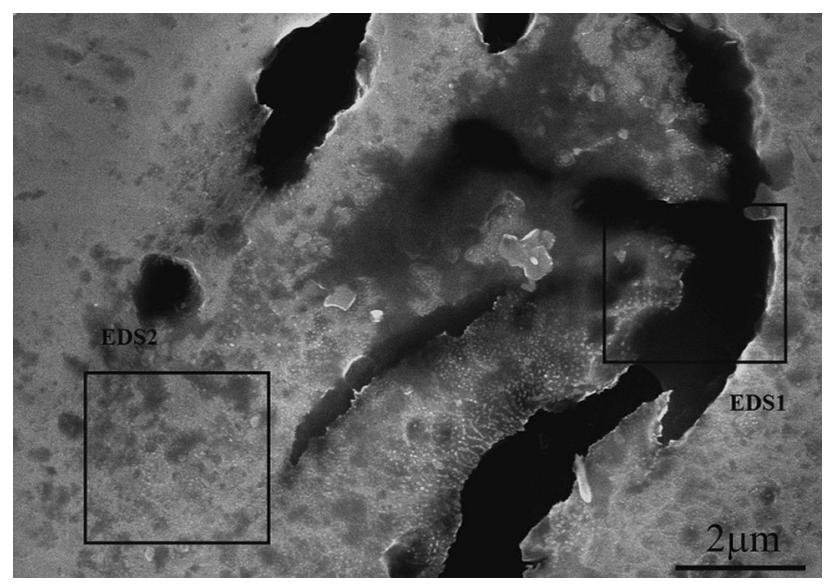

and the other is the state of Ti matrix (such as pores, cracks and interface between filler and matrix). As measured, the in-plane thermal conductivity of graphene can be more than $3000 \mathrm{~W} \mathrm{~m}^{-1} \mathrm{~K}^{-1}$. This can be attributed to the covalent $s p^{2}$ bonding between carbon atoms [21]. Furthermore, the graphene in the interfacial zone provides highly conductive channels for thermal transport and thus facilitates the increase in thermal conductivity of composites. But at the beginning, the number of pores and grain boundary in the composites increase dramatically as graphene added, and this increase causes a sharp rise of heat resistances, especially the $R_{\mathrm{B}}$ rises sharply. According to Eq. (4), the sharp increase of $R_{\mathrm{B}}$ reduces effectively the thermal conductivity of composites $K$. So initially, the thermal conductivity of composites $K$ decreases significantly due to the rise in heat resistances from the pores, cracks and interface between graphene and matrix. At the next stage, along with the continuous increase of grapheme content, the dominant factor of thermal conductivity gradually shifts to be graphene. As the content of graphene increases, the role of an excellent conductive channel for thermal transport appears gradually. Thermal conductivity of composites increases monotonically with graphene increasing from $0.2 \%$ to $0.4 \mathrm{wt} \%$. The transformation process exactly proves that thermal conductivity properties are determined by both graphene content and $\mathrm{Ti}$ matrix (pores, grain boundary and interface between graphene and matrix).

Figure 8 shows the compressive strength of graphene-Ti composites with different graphene contents. The mechanical properties of composites are significantly influenced by addition of graphene. The compressive strength of graphene-Ti composites initially increases drastically as graphene added. The value of compressive strength increases from 1345 to $1595 \mathrm{MPa}$ as graphene adding from 0 to $0.3 \mathrm{wt} \%$. And then, the compressive strength of composites slightly decreases with the addition 

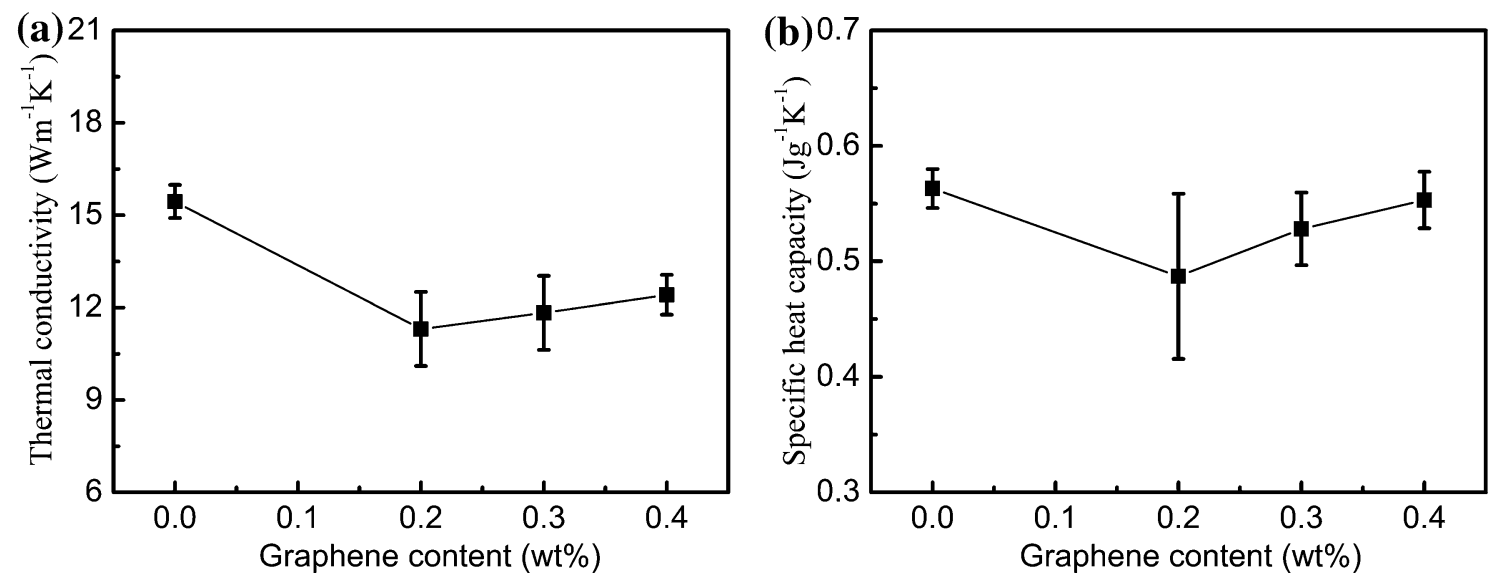

Fig. 7 Thermal conductivity $\mathbf{a}$ and specific heat capacity $\mathbf{b}$ of graphene-Ti composites versus the graphene contents

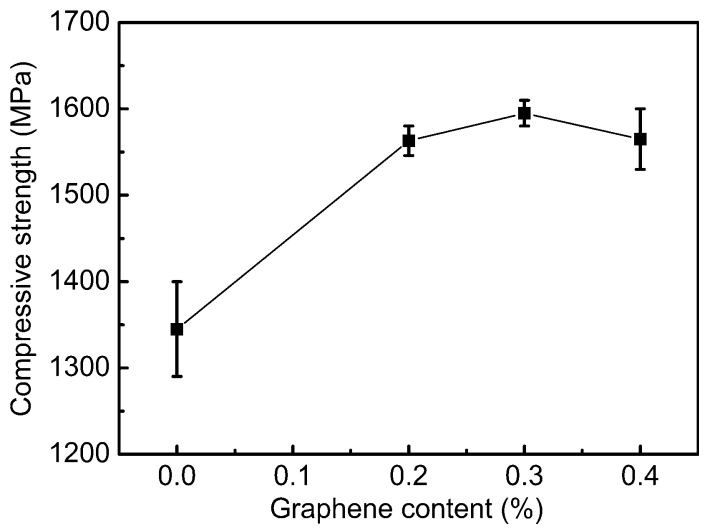

Fig. 8 Compressive strength of graphene-Ti composites with different graphene contents

of $0.4 \mathrm{wt} \%$ graphene. This phenomenon is connected with the fine grain size of Ti matrix by adding graphene and the reinforcing effect of graphene [22]. The compressive strength of graphene-Ti composites can be expressed by the following equation [22],

$\sigma_{\mathrm{c}}=\left(\sigma_{0}+k d_{\mathrm{m}}^{-1 / 2}\right)\left[V_{\mathrm{f}}(\lambda+4) / 4+\left(1-V_{\mathrm{f}}\right)\right]$,

where $\sigma_{c}$ is the compressive strength of the graphene-Ti composites, $\sigma_{0}$ is frictional stress required to sustain dislocation motion in the interior of a grain, $k d_{\mathrm{m}}^{-1 / 2}$ is the grain boundary contribution to yield strength [23], $d_{\mathrm{m}}$ is the average grain size of the Ti matrix, $k$ is recognized as the Hall-Petch coefficient, $V_{\mathrm{f}}$ is the volume fraction of graphene, and $\lambda$ is the aspect ratio of graphene. It is apparent that the compressive strength is affected by the $\lambda, V_{\mathrm{f}}$ and $d_{\mathrm{m}}$. The increase of graphene content reduces the grain size of Ti matrix obviously, which can be observed in Fig. 3 . The fine grain of Ti significantly enhances the compressive strength of composites. On the other hand, with the content of graphene increasing, the graphene is prone to agglomerating at pores (Fig. 6; Table 1), and the aspect ratio of graphene decreases obviously due to the aggregation. According to Eq. (5), decrease in $\lambda$ effectively reduces the compressive strength of composites. Therefore, the variation of compressive strength of composites can be attributed to the interaction effects of the average grain size of Ti matrix $\left(d_{\mathrm{m}}\right)$ and the characteristics of graphene (such as volume fraction $V_{\mathrm{f}}$ and aspect ratio $\lambda$ ) [22].

\section{Conclusion}

The $x$ wt $\%$ graphene-Ti composites $(x=0,0.2,0.3$ and $0.4)$ were synthesized by a powder metallurgy process. The XRD results indicated that the graphene-Ti composites were obtained by microwave sintering, while the impurity phase (titanium carbide, $\mathrm{Ti}_{8} \mathrm{C}_{5}$, Rhombohedral) was also observed. As graphene content increased, the peak intensity of graphene monotonically increased, so did the number of grain boundary and interface between graphene and matrix. But on the contrary, the grain size of composites decreased in the process. The results of EDS showed that the graphene was homogeneously dispersed in Ti matrix by ball-milling process at a certain percentage. The thermal conductivity and specific heat capacity of composites initially decreased drastically with graphene adding, but then increased as graphene increased from 0.2 to $0.4 \mathrm{wt} \%$. This phenomenon can be explained by both the graphene content and the characteristics of $\mathrm{Ti}$ matrix (pores, grain boundary and interface between graphene and matrix). The mechanical properties of composites were significantly influenced by graphene addition. The compressive strength of grapheneTi composites initially increased drastically as graphene added. And then, the compressive strength of composites slightly decreased with the addition of $0.4 \mathrm{wt} \%$ graphene. This phenomenon can be attributed to the interaction effects 
of the average grain size of Ti matrix $\left(d_{\mathrm{m}}\right)$, the volume fraction $\left(V_{\mathrm{f}}\right)$ and the aspect ratio $(\lambda)$ of graphene.

Acknowledgments This work was supported by the Chinese Postdoctoral Science Foundation (No. 2014M561795) and the Postdoctoral Scientific Research Project of Zhejiang Province, China (No. BSH1401037).

\section{References}

[1] A.K. Geim, Science 324, 1530 (2009)

[2] S. Stankovich, D.A. Dikin, G.H.B. Dommett, K.M. Kohlhaas, E.J. Zimney, E.A. Stach, R.D. Piner, S.T. Nguyen, R.S. Ruoff, Nature 442, 282 (2006)

[3] L.M. Veca, M.J. Meziani, W. Wang, X. Wang, F.S. Lu, P.Y. Zhang, Y. Lin, R. Fee, J.W. Connell, Y.P. Sun, Adv. Mater. 21, 2088 (2009)

[4] Y. Cui, L.D. Wang, B. Li, G.J. Cao, W.D. Fei, Acta Metall. Sin. (Engl. Lett.) 27, 937 (2014)

[5] C.L.P. Pavithra, B.V. Sarada, K.V. Rajulapati, T.N. Rao, G. Sundararajan, Sci. Rep. 4, 1 (2014)

[6] J.Y. Wang, Z.Q. Li, G.L. Fan, H.H. Pan, Z.X. Chen, D. Zhang, Scr. Mater. 66, 594 (2012)

[7] M. Rashad, F.S. Pan, A.T. Tao, M. Asif, S. Hussain, J. Gou, J.J. Mao, J. Ind. Eng. Chem. 23, 243 (2015)

[8] L.Y. Chen, H. Konishi, A. Fehrenbacher, C. Ma, J.Q. Xu, H.S. Choi, H.F. Xu, F.E. Pfefferkorn, X.C. Li, Scr. Mater. 67, 29 (2012)
[9] M. Rashad, F.S. Pan, D. Lin, M. Asif, Mater. Des. 89, 1242 (2016)

[10] M. Rashad, F.S. Pan, J.Y. Zhang, M. Asif, J. Alloys Compd. 646, 223 (2015)

[11] K. Kondoh, T. Threrujirapapong, H. Imai, J. Umeda, B. Fugetsu, Compos. Sci. Technol. 69, 1077 (2009)

[12] I. Montealegre Melendez, E. Neubauer, P. Angerer, H. Danninger, J.M. Torralba, Compos. Sci. Technol. 71, 1154 (2011)

[13] Y.H. Zhang, L.J. Yue, L.F. Han, J.L. Chen, D.Z. Jia, F. Li, Comput. Mater. Sci. 56, 95 (2012)

[14] V. Kaushik, A.K. Shukla, V.D. Vankar, Carbon 62, 337 (2013)

[15] F.H. Latief, E.S.M. Sherif, J. Ind. Eng. Chem. 18, 2129 (2012)

[16] L.C. Stearns, M.P. Harmer, J. Am. Ceram. Soc. 79, 3013 (1996)

[17] Y.B. Tao, C.H. Lin, Y.L. He, Energy Conv. Manag. 97, 103 (2015)

[18] S. Wang, M. Tambraparni, J. Qiu, J. Tipton, D. Dean, Macromolecules 42, 5251 (2009)

[19] S. Chatterjee, J.W. Wang, W.S. Kuo, N.H. Tai, C. Salzmann, W.L. Li, R. Hollertz, F.A. Nuesch, B.T.T. Chu, Chem. Phys. Lett. 531, 6 (2012)

[20] K.M.F. Shahil, A.A. Balandin, Nano Lett. 12, 861 (2012)

[21] E. Pop, V. Varshney, A.K. Roy, MRS Bull. 37, 1273 (2012)

[22] F.C. Wang, Z.H. Zhang, Y.J. Sun, Y. Liu, Z.Y. Hu, H. Wang, A.V. Korznikov, E. Korznikova, Z.F. Liu, S. Osamu, Carbon 95, $396(2015)$

[23] Y. Guo, T.B. Britton, A.J. Wilkinson, Acta Mater. 76, 1 (2014) 